\title{
Essai de pathogénie de la peste bovine à la lumière de la conception moderne $\mathrm{du}$ syndrome d'adaptation
}

\author{
Perspectives d'avenir
}

\author{
par G. THIERY
}

Nous avons rapporté, au cours de deux articles précédents, les particularités anatomopathologiques de la peste bovine en fonction du type de virus et de l'espèce affectée, et les caractères hématologiques, histopathologiques et histochimiques de cette affection. Il en résulte un certain nombre de constatations qui permettent de préciser la spécificité des lésions, le mode de transport du virus dans le corps de l'animal, son mode d'action et ses besoins, la défenso que lui opposo l'organisme, enfin le mécanisme de la mort. Après avoir envisagé successivement ces divers points, il sera possible de fixer les conditions que doit remplir un milieu de culture du virus. Par ailleurs, quelques points d'histologie et d'histophysiologie comparée pourront être déterminés.

\section{$*^{*}{ }_{*}$}

\section{Spécificité des lésions.}

Il a été signalé, à l'occasion de l'étude anatomopathologique et histopathologique, que certaines lésions n'étaient pas spécifiques de la peste bovine puisqu'on peut les rencontrer dans d'autres affections : ce sont, notamment, la congestion, l'hémorragie et les ulcérations hémorragiques de la caillette et de la valvule iléo-cæecale chez les bovins et la congestion de l'estomac chez le lapin. Ces diverses lésions s'observent dans les premières phases du syndrome d'adaptation, c'est pourquoi il parait opportun de rappeler les principaux caractères de ce syndrome; il sera dès lors possible d'envisager le déterminisme des phénomènes congestifs et hémorragiques dans l'affection qui nous occupe.

Le syndrome général de l'adaptation correspond à l'ensemble des réactions non spécifiques présentées par un organisme à un agent offensant quelconque. Les réactions perdent leur spécificité par le fait qu'elles sont produites aussi bien par des agents mécaniques, physiques, chimiques que biologiques. Le syndrome évolue en 3 phases plus ou moins différenciées :

- une réaction d'alarme,

- une phase de résistance,

- une phase d'épuisement ou de guérison.

Nous allons, après avoir rappelé les caractères essentiels de chaque phase, rapporter los réactions non spécifiques qu'engendre le virus bovipestique.

La réaction d'alarme se produit dès que l'organisme a été attaqué, et l'on distingue les phénomènes passifs, dits de choc, et les phénomènes actifs de réaction organique, dits de contre-choc.

La phase de choc sera d'autant plus précoce et marquée que le virus s'est disséminé ot dévoloppé plus rapidement dans l'organisme. Elle se traduit, notamment, par une augmentation de la pression artérielle et de la glycémie suivie de leur chute. par de la tachycardie, une vasoconstriction périphérique, et une variation importante de l'équilibre électrolytique sanguin. En même temps que l'on note ces phénomènes, une lésion apparaît. Il s'agit d'ulcérations gastro-intestinalos bien ćtudiécs chez l'homme. L'étude des lésions animales au cours des affections les plus diverses nous a conduit à considérer un certain nombre de lésions comme dues au choc du syndrome d'adaptation. Ce sont, chez les ruminants et les porcins, la congestion parfois extrême de la caillette ou de l'estomac, les ulcérations hémorragiques de la caillette, les foyers congestifs de l'intestin et principalement du gros instestin et du cæcum, avec une localisation particulière : la valvule iléo-cæcale. Dans ce dernier cas, il peut s'agir d'une véritable hémorragie intramuqueuse. Chez le lapin, les ulcérations gastrointestinales sont rares, mais la congestion gastrique 
est habituelle; en outre, on peut déceler une congestion laryngée et souvent trachéale lors de choc très intense.

Nous avons retrouvé, chez les bovins étudiés, les lésions non spécifiques que nous venons d'énumérer tandis que, chez les lapins, la congestion gastrique faisait défaut. On verra que l'état particulier des lapins qui servent aux expériences en est la cause.

A la phase du contre-choc, l'organisme se ressaisit et les divers signes cliniques s'opposent à ce qu'ils étaient au cours de la première phase.

Pendant toute la réaction d'alarme, on note les signes d'un hyperfonctionnement surrénal, d'abord médullaire, ensuite cortical, entraînant une hypertrophie des organes lymphoïdes avec lymphocytose. Les polynucléaires éosinophiles commencent à diminuer de nombre. Ces caractères apparaissent clairement sur les courbes figuratives des variations leucocytaires.

La phase de résistance fait immédiatement suite à la phase de contre-choc. L'organisme s'adapte à son nouvel état, aussi les ulcérations et les foyers de congestion du tube digestif guérissent et disparaissent progressivement tandis que les signes cliniques s'atténuent. C'est pourquoi les lésions non spécifiques peuvent faire défaut, au moins partiellement, lors d'autopsies effectuées sur des animaux morts à la fin de cette phase, lorsque le choc a été peu intense. Pendant cette période, les organes lymphoïdes s'atrophient et involuent très légèrement. Il en résulte une lymphopénie, cependant qu'apparaissent une polynucléose neutrophile et une polynucléopénie éosinophile. Ces caractères hématologiques sont très nets, aussi bien chez les bovins que chez les lapins, mais la lymphopénie dépasse de beaucoup ce que l'on observe lors d'un syndrome d'adaptation banal. Ici se trouve donc extériorisée l'action destructive du virus vis-à-vis des lymphocytes. De même, la lésion des follicules lymphoïdes domine et cache la discrète atrophie des ganglions.

A cette phase peuvent faire suite deux états opposés : ou bien l'organisme a mis à contribution toutes ses réserves défensives et les a épuisées, c'est la phase d'épuisement qui se termine par la mort; elle est la règle chez les bovins; ou bien l'organisme domine l'agent offensant et il guérit, c'est ce que l'on observe chez les lapins.

Ainsi, des considérations de pathologie générale conduisent à la conclusion que certaines lésions sont dénuées de spécificité. Ce point est encore confirmé lorsque l'on cherche le déterminisme intime de ces phénomènes d'origine vasculaire.

En effet, sous l'action du virus au cours de la réaction d'alarme, la stimulation cortico-surréna- lienne se traduit par une décharge de glycocorticoïdes. Ceci est prouvé par la disparition des polynucléaires éosinophiles, les variations des autres leucocytes et enfin la surcharge glycogénique du foie à cette phase. La sécrétion des hormones cortico-surrénaliennes entraîne une dépolymérisation de certains polysaccharides, d'où expulsion des grains de mastocytes. Il se produit alors une libération locale et simultanée d'héparine et d'histamine. Ces substances agissent directement sur les petits vaisseaux et produisent une vasodilatation simple, ou une vasodilatation extrême compliquée parfois d'hémorragies. C'est ce que l'on observe dans la caillette des bovins, organe particulièrement riche en mastocytes ; c'est ce que l'on décèle également au niveau de la valvule iléo-cæcale de ces mêmes animaux : lorsque les lésions sont apparues, le nombre des mastocytes a diminué. Il est intéressant de noter d'autre part que la muqueuse de l'intestin grêle renferme un très grand nombre de polynucléaires éosinophiles. On a vu qu'ils proviennent du torrent circulatoire dont ils sont sortis à l'occasion de la réaction d'alarme. Il est probable que l'infestation parasitaire du tube digestif est responsable déjà d'une accumulation de polynucléaires éosinophiles dans le chorion muqueux intestinal. Il existe un véritable antagonisme entre les polynucléaires éosinophiles et les mastocytes dans l'intestin. Quand les polynucléaires éosinophiles sont présents, les mastocytes ne libèrent pas leurs granulations et il n'y a pas de trouble vasculaire. L'absence de polynucléaires éosinophiles dans la caillette et la valvule iléo-cæcale semble bien confirmer ce point de vue.

Nous avons cherché à prouver le rôle de l'histamine dans ces lésions par l'injection de fortes doses d'un antihistaminique à des bovins affectés de peste bovine afin d'inhiber les phénomènes congestifs et hémorragiques du tube digestif. Nous avons traité un animal gravement infecté à l'aide de Phénergan. Après un sommeil de 36 heures au cours duquel la température est tombée de $41^{\circ} \mathrm{C}$ à $36,2^{\circ} \mathrm{C}$, 1'animal n'a plus présenté qu'une très faible réaction thermique de 24 heures $\left(38,5^{\circ}\right)$ avant de reprendre une température normale. L'animal a guéri et nous $n$ 'avons pu en faire l'autopsie tandis que les témoins sont morts. Nous aurions voulu recommencer cette expérience, mais cela nous a été pour l'instant impossible.

On peut se demander si le Phénergan, en dehors. de son action directe sur l'histamine, n'a pas joué dans l'organisme un rôle inhibiteur de la division cellulaire comme cela est observé chez les végétaux (I). Cette constatation, si elle était confirmée, serait d'une grande importance car le virus, comme on le verra, a un besoin important d'acides 
nucléiques. De plus, sa portée dépasserait certainement le cadre de la peste bovine.

Il est habituel d'observer chez le lapin une gastrite hémorragique. Nous ne l'avons pas constaté, mais nos animaux ont reçu pendant un certain temps un excès de vitamine $D_{2}$ dont l'action semblable à celle de la cortisone est bien connue. Il en est résulté un empêchement de la mise en réserve des granulations que renferment les mastocytes, notamment dans l'estomac, comme nous l'avons contrôlé. Par ailleurs, les conditions thermiques entraînent des stress répétés de l'organisme qui aboutissent au même résultat. L'examen histologique des surrénales des témoins nous a toujours montré des glandes à grande activité, ce qui permet de comprendre la différence de nos résultats par rapport aux notions classiques.

\section{$2^{\circ}$ Transport du virus dans l'organisme.}

Il est admis depuis longtemps que le virus est véhiculé dans tout l'organisme par le sang. L'histopathologie confirme cette manière de voir chez le lapin car, indépendamment du fait que le sang soit virulent, on constate l'absence constante de lésion des points lymphoídes non vascularisés alors qu'ils drainent la lymphe.

On sait actuellement que, dans le sang, le plasma n'est pas virulent, mais que les hématies le sont très peu tandis que les leucocytes le sont fortement (2-3).

Il ne semble pas cependant que l'on ait déterminé quelle était la variété de leucocyte responsable du transport du virus. Or par l'étude des lésions, il apparait qu'en dehors des phénomènes d'adsorption du virus sur les globules rouges et divers globules blancs, celui-ci est activement transporté par les polynucléaires.

Lors de l'examen des lésions, et ceci est toujours très net lorsqu'on les étudie avec la méthode de Baker sur coupes à congélation, on constate que l'arrivée du virus dans un tissu coïncide avec l'apparition des polynucléaires neutrophiles (ou pseudo-éosinophiles chez le lapin). Ceci laisse supposer que le virus est supporté par cette variété de polynucléaires et ainsi disséminé dans l'oŕganisme. On pourrait être tenté de séparer les polynucléaires des autres leucocytes pour prouver, par inoculation à des animaux neufs, qu'ils supportent bien le virus, mais, en raison des phénomènes d'adsorption sur les divers globules sanguins, il faudrait déterminer la dose minima infectante de chaque type de cellule. Toutefois, indépendamment de cette démonstration directe, un certain nombre d'arguments sont en faveur de cette thèse.

L'étude des tableaux de la dose minima infectante
(D.M.I.) par organe, établie antérieurement au laboratoire chez le lapin (4), montre bien la relation qui existe entre la teneur en polynucléaires des organes considérés et la D.M.I. Pour la rate, qui n'est infiltrée que de très peu de polynucléaires, la D.M.I. est de $0,1 \mathrm{mg}$ alors que pour les ganglions mésentériques, qui en renferment un très grand nombre, elle est de $0,004 \mathrm{mg}$. La D.M.I. du poumon et du foie est proportionnelle à la teneur des organes en sang donc en globules blancs. Il ne s'agit pas d'un support par les globules rouges car la rate, qui présente une D.M.I. voisine de celle du sang, est moins infectante que les ganglions mésentériques, qui n'en renferment pratiquement pas. Ces quelques considérations montrent encore que les lésions des follicules lymphoïdes paraissent d'autant plus virulentes que l'infiltration par les polynucléaires a été plus intense puisque certains tissus comme le foie et le sang renferment du virus et ne présentent pas la moindre lésion nécrotique autre que celle des polynucléaires circulants.

Nous avons, par ailleurs, cherché à éliminer les polynucléaires neutrophiles de l'organisme du lapin à l'aide de moutarde à l'azote (le benzène s'est révélé, après 2 semaines d'intoxication, inactif sur nos lapins et nous n'avons pas encore eu la possibilité de les irradier aux rayons $\mathrm{X}$ ou de les traiter à l'uréthane). Nous avons constaté, après le traitement à la moutarde à l'azote d'origine américaine, une chute importante des leucocytes circulants, intéressant principalement les polynucléaires neutrophiles. Il en est résulté un retard d'au moins 24 heures au début de la poussée thermique. Par ailleurs, l'infiltration par les polynucléaires des follicules a été très atténuée. Cette atténuation correspond essentiellement au nombre des follicules. affectés (les $2 / 3$ de l'atteinte des témoins) tandis que le stade final de la lésion était pratiquement inchangé. Nous avons pu constater également, sur ces animaux, une augmentation des lésions épithéliales; les inclusions cytoplasmiques amygdaliennes étaient plus nombreuses. Il semble que l'on ait modifié le tropisme du virus et que, de lymphoréticulotrope, il tende à redevenir épithéliotrope. Par passages répétés dans ces conditions, il serait peutêtre possible de lui rendre sa virulence pour les taurins. L'emploi de moutarde à l'azote d'origine française (Caryolysine) n'a produit qu'une chute réduite du nombre des polynucléaires circulants; la poussée thermique n'a été retardée que d'une manière très discrète. Ceci montre bien le rapport qui existe entre la présence des polynucléaires circulants et le développement de l'affection.

La liaison du virus et des polynucléaires ne doit pas surprendre puisque dans le sang, si l'on met à part les phénomènes physiques d'adsorption, les 
polynucléaires neutrophiles jouent le rôle de microphagocytes, c'est-à-dire phagocytant les particules microscopiques et inframicroscopiques.

Ainsi, le virus semble bien être, au moins chez le lapin, et sans doute également chez les bovins, transporté par les polynucléaires neutrophiles. Cette affinite du virus pour ces cellules est intéressante à considérer car on peut déjà entrevoir un mode de production du virus d'un rendement accru ainsi que nous pourrons le voir lorsque l'on connaîtra les besoins du virus.

\section{$3^{\circ}$ Mode d'action et besoins du virus.}

Le virus, qu'il s'agisse des bovins ou des lapins, agit sensiblement de la même manière sur les cellules; or on sait que le virus induit la cellule à faire sa synthèse ou au moins celle de ses constituants, si bien que la connaissance du mode d'action d'un virus ouvre quelques aperçus sur sa constitution.

Le virus, au niveau des épithéliums, entraîne une multiplication des cellules et plus particulièrement des noyaux, au point d'engendrer des plasmodes dont le rapport nucléoplasmatique est considérable par rapport aux cellules qui sont à son origine. Cette stimulation rappelle celle de l'hétérochromatine vis-à-vis des mitoses. Le virus conduit ainsi la cellule à fabriquer des noyaux, donc des acides nucléiques du type désoxyribonucléique. Par ailleurs, nous avons constaté la consommation importante de l'acide ribonucléique au début de la formation des lésions. Ces deux remarques laissent supposer que le virus a besoin de ces deux acides, ce qui n'est pas pour nous surprendre puisque l'on sait qu'ils sont indispensables à la synthèse protéique; or, les virus, en général, sont de nature protéique.

La différence de comportement du virus selon les divers animaux vient essentiellement du fait qu'il attaque des ribonucléoprotéines différentes. Chez les taurins, il paraît utiliser avec prédilection les nucléoprotéines des épithéliums malpighiens; chez les zébus, il dégrade en outre celle des lymphocytes dans une zone particulière du tissu lymphoïde; chez les lapins, il abandonne pratiquement les épithéliums pour le tissu lymphoïde.

La zone lymphoide où le virus vient se localiser est en effet particulière; c'est le centre des follicules lymphoïdes, là où est synthétisé l'acide ribonucléique tandis qu'à la périphérie sont apparemment synthétisées les gamma-globulines. C'est pourquoi la maladie engendrera d'autant plus d'anticorps que les follicules ne sont pas complètement détruits, et l'on peut se demander si l'immunité du lapin à une nouvelle infection par le virus lapinisé est due aux anticorps ou à l'absence de matériaux propres à son développement. Il semble que la deuxième hypothèse soit valable comme nous allons le montrer avec l'aide de la cortisone.

Cette notion de tropisme du virus lapinisé pour les follicules lymphoïdes est utilement emiployée pour la vaccination des taurins chez lesquels le virus bovipestique naturel attaque essentiellement les épithéliums. Notons cependant que, chez les taurins de Côte-d'Ivoire, la réaction lymphoïde au virus bovipestique est plus accusée que chez les taurins d'autres régions. C'est ce qui explique sans doute la forte réaction vaccinale de ces animaux lors do l'injection de virus lapinisé.

On pourrait dès lors se dire que le virus lapinisé va tuer le zébu chez lequel le virus naturel est à la fois épithéliotrope et réticulolymphotrope et devient même, par passages répétés, fortement réticulo-lymphotrope. Mais nous avons yu, et cette particularité augmente avec les passages, que le zébu fabrique des inclusions cytoplasmiques dans ses follicules. Il s'agit ici d'un mode de défense de l'organisme. Le virus lapinisé, presque exclusivement réticulo-lymphotrope, va trouver chez le zébu une défense importante qui ne lui laissera pas le temps de s'adapter mais permettra cependant la production des anticorps. Ceci montre encore l'intérêt de ce virus adapté pour la vaccination des animaux car il se localise directement dans les lieux où sont élaborées la majeure partie des gammaglobulines.

Nous avons cherché à déterminer le rôle des lymphocytes dans la maladie. Pour cela, nous avons imprégné l'organisme de lapins par la' cortisone (10 cg par jour pendant 3 jours), puis nous avons injecté le virus lapinisé en continuant le traitement cortisonique pendant encore 2 jours. Le stéroïde a entraîné une involution des formations lymphoïdes ainsi que diverses viciations métaboliques. Les animaux infectés ne présentent, lorsqu'elle existe, qu'une très discrète réaction thermique. Ils paraissent réfractaires. L'examen histologique montre une déshabitation assez prononcée de tous les follicules lymphoides sans la moindre lésion classique du virus. Il semble que le virus n'ait pas trou'vé dans l'organisme les éléments indispensables à sa multiplication. Ainsi se manifeste le rôle, au moins pour le virus lapinisé, des substances élaborées dans le centre germinatif des follicules lymphoïdes, par les cellules de la lignée lymphoíde, puisque le réticulum est inchangé. Il ne semble pas que l'on doive incriminer la seule variation des possibilités phagocytaires des leucocytes' (dont les polynucléaires) produites par la cortisone, puisque le virus introduit directement dans le sang est disséminé dans tout l'organisme. 
Ainsi, un excès de cortisone inhibe le développement du virus, tandis qu'une substance à action voisine, comme la vitamine $\mathrm{D}_{2}$, semble responsable d'une plus grande intensité des lésions. Il est vrai que son administration correspond à une dose très faible, appliquée pendant longtemps, de stérolde. L'organisme y est adapté et subit moins intensément les réactions non spécifiques du stress viral.

A l'inverse de cette action hormonale, nous avons traité des lapins par l'hormone somatotrope (5 UI par jour) pendant les jours qui ont précédé l'inoculation du virus et pendant l'évolution de la maladie. Nous n'avons pas noté de différence significative par rapport aux témoins non traités.

Dans les lésions des follicules lymphoïdes, principalement chez le lapin, il y a envahissement de la zone centrale par les polynucléaires neutrophiles. Cet appel est dû apparemment, lorsque la lésion a débuté, aux produits de désintégration cellulaire et plus particulièrement aux nucléoprotéines. Par la suite, les polynucléaires agissent par leurs réserves enzymatiques, notamment les enzymes protéolytiques qui entraînent la fluidification des débris cellulaires d'où le nettoyage rapide des follicules affectés. Le tropisme du virus pour les polynucléaires ne paraît pas fortuit ; il semble dû justement à la préscnce des onzymes ct l'on sait que les polynucléaires sont parmi les leucocytes ceux qui en supportent la plus grande variété et que, de plus, ils possedent en abondance les enzymes protéolytiques. Le virus a donc à sa disposition, au sein des lésions, les ferments qui lui sont nécessaires.

Nous avons mentionné au début de cet exposé que le mauvais état des animaux peut jouer un rôle sur le développement de la maladie. Nous avons effectivement constaté qu'en général les lésions sont beaucoup moins intenses chez les sujets hydrocachectiques. Il semble que le virus ne trouve plus en quantité suffisante les substances qui lui sont indispensables.

Ainsi, parmi les besoins du virus, on peut mentionner au premier chef les acides nucléiques, sans que l'on puisse déterminer directement les rapports de l'un et de l'autre. Dans les lésions, la consommation d'acide ribonucléique est importante et nous nous permettrons d'insister encore sur ce point. Ce type d'acide joue, en effet, un grand rôle dans la synthèse des protéines en général. Notons également que les cellules qui en renferment beaucoup ct qu'attaque le virus ont un métabolisme actif et que le métabolisme normal est vicié en faveur d'une synthèse d'acide désoxyribonucléique puisque les divisions cellulaires deviennent fréquentes. Bien plus, des organes comme la caillette présentent une intense stimulation mitotique, même lorsque les lésions spécifiques sont très discrètes. Le métabolisme des acides désoxyribonucléiques dans cet organe serait alors très important et correspondrait à une intense production de virus puisque Jacotot (5) montre que cette portion du tube digestif est plus riche en virus que les autres tissus de l'organisme. Ceci tendrait à prouver que les besoins du virus en acide désoxyribonucléique sont très grands et que le rôle de l'acide ribonucléique n'est que secondaire. Quoi qu'il en soit, les deux types d'acides nucléiques paraissent indispensables au virus bovipestique. Par la suite, au moins pour les virus lapinisé et caprinisé, certaines substances élaborées par les lymphoblastes et les grands lymphocytes lui sont nécessaires, sans que l'on puisse affirmer qu'elles diffèrent des acides précédents. Le tropisme du virus pour les polynucléaires neutrophiles laisse prévoir que les enzymes protéolytiques lui sont très utiles.

\section{Défense de l'organisme vis-à-vis du virus.}

Il semble que l'organisme subisse l'action du virus, aussi bien au niveau des épithéliums que des follicules lymphoides, sans présenter, bien souvent, de réactions de défense. Il est difficile d'interpréter la stimulation mitotique comme un moyen de défense opposé par la cellule qui utiliserait pour elle les acides désoxyribonucléiques. Il paraît plus probable que les acides nucléiques du virus agissent directement sur les cellules à la manière de l'hété rochromatine. Cependant, la formation des inclusions cellulaires cytoplasmiques traduit une édification des cellules. On a vu le rôle que jouent à la fois le chondriome et l'appareil de Golgi dans lour ćlaboration. Il est toutefois difficile de préciser comment l'inclusion agit sur le virus. Est-ce pour l'inactiver, est-ce pour l'enrober d'une substance qui l'isole ou le neutralise? Cette dernière hypothèse parait le mieux correspondre à la réalité. On sait, en effet, par l'étude d'autres inclusions cellulaires à l'aide du microscope électronique qu'elles renferment le virus; d'autre part, il cst oncore virulent car l'injection d'inclusions dans un organisme neuf peut reproduire la maladie. Quoi qu'il en soit, il s'agit d'une réaction heureuse puisqu'une cellule qui renferme des inclusions paraît survivre au moins un certain temps, tandis que celle qui est atteinte sans faire d'inclusions dégénère et meurt rapidement.

Nous avons mentionné la présence des histones, aussi peut-on se demander si ces substances ne jouent pas un rôle inhibiteur ou isolant vis-à-vis du virus. Elles ont de toute façon une action heureuse. Aussi est-il tentant de préconiser l'administration d'histones ou des substances qui en assurent la 
genèse, mais on ignore encore le métabolisme de ces substances dans l'organisme.

On constate en général, dans les formes d'évolution lente, un plus grand nombre d'inclusions cellulaires que dans les formes rapides, ce qui traduit une plus grande défensc de l'organisme; la virulence est moindre puisque, dans la lutte entre l'organisme et le virus, ce dernier est partiellement neutralisé.

\section{$5^{0}$ Cause de la mort lors de peste bovine.}

La cause déterminante de la mort est difficile à préciser, car dire que la mort est due au virus cache notre ignorance. L'étude cytologique du sang ne permet pas de noter la moindre anomalie pouvant entraîner l'arrêt des fonctions vitales. Les modifications du nombre des leucocytes n'entraînent pas, habituellement, à elles seules, une mort aussi rapide, d'autant plus qu'elles sont semblables chez le lapin et le bovin et que le premier animal survit à l'infection. L'examen des courbes électrophorétiques du sérum sanguin n'apporte aucun élément.

Il ne semble pas s'agir d'une intoxication car les muscles, les endothéliums, les surrénales n'en présentent pas les stigmates. Il est vrai qu'il n'a pas encore été mis en évidence une toxine due à ce virus.

Nous avons signalé, au cours de l'étude de la maladie, une congestion de la substance grise nerveuse, tant du névraxe que de l'encéphale, avec dégénérescence de cellules nerveuses. Il est possible de rendre ces lésions responsables de l'arrêt d'organes vitaux tels que le cœur mais rien ne permet d'affirmer que l'on est en présence d'une cause unique.

Il a été mentionné l'état habituellement amaigri des cadavres. Il traduirait l'épuisement de l'organisme et serait pour Curasson (6) la cause habituelle de la mort. L'amaigrissement des malades résulte essentiellement d'une perte de protéines, mais aussi d'une légère déshydratation du secteur intracellulaire de l'organisme. La perte protéique s'explique aisément par la consonmation de l'acide ribonucléique au moment de la formation des lésions. La déshydratation cellulaire se traduit par une faible réduction de taille des cellules des divers tissus épithéliaux, dont le rein. Elle est la conséquence du déséquilibre électrolytique résultant de la décharge en stéroïdes surrénaliens au cours du syndrome d'adaptation. On se trouve ainsi en présence de deux facteurs importants car ils conditionnent la vie des cellules et par cela même la vie de l'organisme entier. On verra que les réactions dues aux stéroïdes surrénaliens diffèrent nettement chez les bovins et chez les lapins, c'est ce qui paraît expliquer la survie de ce dernier animal lors d'infection par le virus lapinisé.

Ainsi, les bovins semblent décéder à la suite, d'une part, d'une impossibilité de synthétiser rapidement les protéines vitales; d'autre part, d'un déséquilibre électrolytique humoral grave. Le dernier trouble est aggravé par la diarrhée. On doit en outre tenir compte des lésions nerveuses qui, lorsqu'elles sont graves, peuvent expliquer certains accidents mortels.

\section{$6^{\circ}$ Conséquences histologiques et histophysiolo- giques.}

Il peut paraître paradoxal de tirer des enseignements histologiques et histophysiologiques de l'étude de lésions. Néanmoins, il est possible de déceler certaines particularités lors de la réparation de ces lésions. Bien plus, la spécificité de l'attaque cellulaire de certains virus permet d'identifier, mieux qu'une réaction histochimique, la présence de certaines substances. La détermination par le virus paraît même plus fine et plus spécifique que les grossières réactions chimiques dont on dispose actuellement.

On a vu que le virus bovipestique naturel oú lapinisé attaque dans les ganglions les follicules lymphoïdes, mais respecte les cordons médullaires. Ceci parait bien montrer la différence entre les cellules des follicules et celles des cordons médullaires malgré une morphologie semblable. Le virus montre mieux qu'une réaction chimique la différence de fonction de ces deux types de cellules. Lorsque, chcz le lapin, les follicules lymphoïdes ont disparu, la lymphopoïèse est extrêmement réduite, elle est nulle dans certains ganglions malgré la présence de cordons médullaires morphologiquement normaux.

On peut faire une remarque analogue au niveau des plaques de Peyer chez le lapin. La zone lymphoïde suprafolliculaire n'assure pas non plus de suppléance. Elle semble très proche des cordons médullaires des ganglions.

Le virus attaque de la même façon les lymphocytes et les thymocytes. Cet argument est en faveur d'une commune origine, théorie admise actuellement par la majorité des auteurs.

Les corpuscules de Malpighi ne sont que très rarement affectés. S'agit-il d'une conséquence vasculaire ou d'une différence histophysiologique? Il est difficile de trancher la question mais la possibilité de lésion est plutôt en faveur de la première hypothèse.

Il est intéressant de noter encore que le virus qui possède à la fois un lymphotropisme et un épithéliotropisme engendre les plus belles lésions dans un tissu lymphoépithélial, à savoir l'amygdale. 
La réaction sanguine d'adaptation au virus est analogue chez le lapin et les bovins, mais à une échelle de temps différente, ce qui montre bien la lenteur des réactions du bovin par rapport au lapin. Or, la surrénale du lapin est riche en lipides figurés tandis que celle du bovin renferme en abondance des lipoproténes et très peu de lipides figurés. Cette lenteur de réaction existe d'ailleurs chez les bovins à l'égard des agressions les plus diverses. Cette constatation semble indiquer que les précurseurs des 11 oxycétostéroïdes sont plus facilement métabolisés chez les lapins que chez les bovins. Rappelons à ce propos, comme nous l'avons montré avec Drieux, Houdinière et Mlle Dassieu (7), que l'urine des bovins hydrohémiques est particulièrement riche en stéroïdes surrénaliens. Il y a peut-être là l'explication des réactions anormales des bovins en mauvais état à l'infection par le virus bovipestique, analogues à ce que nous avons provoquć chez le lapin par la cortisone.

De plus, la rapidité de la réaction surrénale peut encore conditionner le type de lésion. Chez le lapin, la surrénale réagit précocement et l'on note un tropisme réticulolymphocytaire; chez les bovins. la réaction surrénalienne est lente et progressive et le tropisme épithélial est primitif. La libération par l'organisme lui-même des stéroïdes surrénaliens ne produit qu'une involution très discrète des follicules lymphoïdes au moment où le virus est présent. Il profite donc de l'involution des follicules tandis que l'injection préventive de cortisone à forte dose entraîne une involution lymphoïde accusée et terminée lorsque le virus est introduit dans l'organisme, d'où la différence d'action d'une même hormone selon la dose et la période d'activité. Le même phénomène a été observé chez un lapin qui présentait un adénome surrénalien. L'infection a entrainé une surrénalite hémorragique et des lésions des follicules lymphoïdes particulièrement intenses.

Notons également que, chez les taurins de Côted'Ivoire, les surrénales paraissent plus actives que chez les taurins d'autres régions. En effet, les lésions non spécifiques sont plus intenses, ce qui traduit une réaction d'alarme plus accusée avec forte décharge de glucocorticoïdes. Comme nous l'avons signalé, les lésions lymphoïdes sont plus importantes. ce qui est en accord avec ce que nous venons de rapporter à propos du parallélisme de l'activité de la surrénale et de l'intensité des lésions lymphoïdes.

L'étude de la peste bovine permet donc de considérer que la lymphopoïèse est le fait des seuls follicules lymphoïdes et qu'à l'état normal, il ne peut y avoir de suppléance par les cordons médullaires des ganglions ou les zones suprafolliculaires intestinales. On doit donc considérer comme possible l'existence d'un facteur spécial responsable de la transformation des cellules réticulaires en lymphoblastes comme cela se rencontre au cours de certains états pathologiques. Par ailleurs, la composition chimique différente des lipides surrénaliens chez les bovins et les lapins conditionne la rapidité et l'intensité des réactions au cours du syndrome d'adaptation. Mentionnons encore la présence de petites sphérules acidophiles que l'on rencontre dans la zone glomérulée de la surrénale des bovins et jamais chez les lapins. On peut se demander s'ils ne participent pas à l'élaboration d'hormones particulières, de nature peut-être protéique, intervenant dans la différence de comportement aux agressions de cette espèce de ruminants.

\section{$*^{*} *$ \\ Perspectives d'avenix}

Il a été mentionné, parmi les besoins du virus bovipestique, les acides nucléiques: On pouvait, dès lors, être tenté d'agir sur lui par l'intermédiaire de ces substances. N'ayant pas les animaux d'expérience à notre disposition, nous nous sommes borné à traiter un autre virus qui paraît, lui aussi, nécessiter à la fois des ribonucléoprotéines et des désoxyribonucléoprotéines : le virus rabique. Nous avons ainsi, au cours d'une première série d'expériences (8), mis en contact à la température de $37^{\circ}$ pendant 2 heures le virus rabique et une solution de nucléinate de sodium. Le virus ainsi traité a conservé chez la souris sa virulence normale par voie intracérébrale tandis que certaines souches montrent, à une dilution suffisante, une virulence très atténuée par voie intramusculaire. Actuellement, nous poursuivons des recherches dans ce sens afin de déterminer jusqu'a quel point on peut transformer le virus rabique. Il paraît intéressant d'entreprendre des recherches analogues avec le virus bovipestique, ce qui permettrait peut-être d'obtenir un virus de propriétés vaccinales particulières.

Les acides nucléiques étant nécessaires au virus, il semble que l'on pourrait agir sur lui, dans l'organisme malade, par l'injection d'acides nucléiques viciés ou isolés d'une autre espèce animale qui pourraient dévier le métabolisme du virus, ou d'acides nucléiques radioactifs qui pourraient agir directement sur lui.

Par ailleurs, l'étude des besoins du virus permet d'envisager une amélioration de sa production, aussi bien pour le virus bovipestique naturel que pour le virus lapinisé. Il suffirait, en effet, de fournir au virus les acides nucléiques qui lui sont nécessaires et qui possèdent la propriété de faire un appel 
de polynucléaires neutrophiles pour réaliser un milieu de culture vivant chez l'animal d'expérience. On peut y parvenir de la manière suivante :

On injecte une suspension stérile d'acides nucléiques dans le péritoine du sujet destiné à la production du virus. L'injection a lieu le troisièrne jour suivant l'infection chez les bovins, 24 heures après celle-ci chez les lapins. Il en résulte un appel de polynucléaires neutrophiles qui, à ce moment, sont pour un grand nombre chargés de virus. I. péritonite purulente aseptique, quant aux bactéries, est un excellent milieu de culture pour le virus bovipestique car elle renferme les acides nucléiques indispensables au virus et les polynucléaires avec leurs enzymes protéolytiques. La récolte aseptique du liquide virulent peut, dès lors, s'effectuer d'une manière particulièrement simple.

\section{$* *$}

\section{Conclusion}

L'étude des lésions permet de supposer que le virus est transporté par les polynucléaires neutrophiles. La recherche de ses besoins montre le rôle important joué par les nucléoprotéines dans sa multiplication, aussi est-on autorisé à envisager une amélioration dans sa production par la réalisation d'un véritable milieu de culture dans un organisme vivant. Il reste néanmoins à contrôler sa valeur par l'cxpćrimentation et, s'il se révèle satisfaisant, nous aurons atteint le but que nous nous sommes proposé en entreprenant ce travail.

(Travail du Laboratoire Fédéral de l'Elevage « G. Curasson ». Directeur : P. Mornet).

\section{BIBLIOGRAPHIE}

(1) DEYSSON (G.), DECOURT (P.) et ANGUERA (G.). - Action de la chlorpromazine (4560 RP) comparée à celle de diverses autres substances sur la division des cellules végétales. Contribution à l'étude de l'action narcobiotique. Rev, Path. géné. et comp, 1954, 54, 868-883.

(2) TOOD (C.) et WHITE. - Experiments on Cattle Plague. Hyg. Int. Depart. of Public. Health. Govt. Press, Le Caire, 1914.

(3) HORNBY. - The Distribution of Rinderpest Virus in Infected Blood. J. of Comp. Path. and Therap., 1928, 41, 17-24.

(4) MORNET (P.), ORUE (J.), LABOUCHE (C.), MAINGUY (P.) et MAHOU (R.). - Les virusvaccins contre la peste bovine. Le virus bovipestique lapinisé. Revue Elevage et Méd. Vét. Pays Tropicaux, 1953, 6, 125-166.

(5) JACOTOT $\left(\mathrm{H}_{\text {. }}\right.$. - - Sur la teneur en virus de quelques tissus de veaux atteints de peste bovine expéximentale. Bull. SoC' Path. Exot., 1931, 24, 21-26.

(6) CURASSON (G.). - Traité de pathologie exotique vétérinaire et comparée. Vigot Frères, Editeurs, Paris, 1942, tome 1.

(7) DRIEUX (H.), THIERY (G.), HOUDINIÈRE (A.) et DASSIEU (G.). - Recherches sur les viandes dites hydrohémiques. $15^{\mathrm{\theta}}$ Congrès Intern. Vét:, Stockholm 1953, 2, 1151-1155.

(8) THIERY (G.). - Premiers résultats de l'étude de l'action de diverses hormones et du nucléinate de sodium sur le virus rabique. C.R. Acad. Sciences, 1956, 242, 945-947.

\section{SUMMARY}

The pathogenicity of rinderpest in the light of recent knowledge of the adaptation syndrome.

Future prospects

The results of experiments carried out on the adaption syndrome have shown that the number of specific lesions in rinderpest can be reduced. Neutrophils seem to be very important in the transport of virus throughout the body. The virus requirements mainly consist of dcsoxyribonucleic acids followed by ribonucleic acids and sometimes proteolytic enzymes. Specific cellular inclusions must be regarded as defence reactions against the virus. The occurrence of death seems partly to be due to the destruction of ribonucleo-proteic acids but mainly to a humoral electrolytic imbalance. Histophysiology of lymphoid tissue and adrenal glands is also described. I living culture medium can be obtained from cattle under experimental conditions. 


\section{RESUMEN}

Ensayo sobre la patogenia de la peste bovina en vista de la modema concepción del sindrome de adaptación. Perspectivas del futuro

El estudio del sindrome general de adaptación en animales receptivos al virus de la peste bovina, permite limitar el número de lesiones especificas. El transporte del virus en el organismo parece sea activamente realizado por los polinucleares neutrófilos. Entre las necesidades del virus hay que citar en primer lugar los ácidos desoxiribonucleicos, después los ácidos ribonucleicos y, accesoriamente, los enzimas proteolitiços. El organismo se defiende contra el virus por la elaboración de inclusiones celulares especificas. Parece ser que la muerte sea la consecuencia de una destrucción de lós ácidos ribonucleoproteicos y sobre todo de un desequilibrio electrolitico humoral. El autor precisa algunos puntos de histofisiologia de los organos linfoides y de las suprarrenales de los animales estudiados. Se precisan, finalmente, las condiciones de realización de un medio de cultivo vivo en el animal de experimentación. 